Pacific Journal of Mathematics

AN IMBEDDING THEOREM FOR INDETERMINATE 


\title{
AN IMBEDDING THEOREM FOR INDETERMINATE HERMITIAN MOMENT SEQUENCES
}

\author{
Francis J. NARCOWICH
}

\begin{abstract}
Hermitian moment sequences are generalizations of classical power moment sequences to bounded operators on a Hilbert space. The main result is that every indeterminate Hermitian moment sequence on a complex Hilbert space can be imbedded in a determinate Hermitian moment sequence on an enlarged Hilbert space in the sense that the first sequence is a compression of the second. This implies the existence of determinate Hermitian moment sequences which, when compressed, are indeterminate and leads to the following questions: Which orthogonal projections on the Hilbert space give rise to determinate compressions of a fixed, determinate sequence? What structure do these projections induce on the underlying Hilbert space?
\end{abstract}

Background. Let $\mathscr{H}$ be a complex Hilbert space, with inner product $(\cdot, \cdot)$ and norm $\|\cdot\|$, and let $B(\mathscr{H})$ be the set of all bounded linear operators on $\mathscr{H}$. A sequence $\left\{T_{j}\right\}_{j=0}^{\infty}, T_{j} \in B(\mathscr{H})$, is said to be an Hermitian moment sequence (cf. J. S. MacNerney [4]) if there exists a positive operator-valued measure (cf. Berberian [2]), $\mu(\cdot)$, defined on the Borel sets of $(-\infty, \infty)$, such that

$$
T_{1}=\int_{-\infty}^{\infty} t^{\prime} d \mu(t), \quad j=0,1,2, \cdots .
$$

Necessary and sufficient conditions for a sequence of operators to be of the form (1) and, in addition, be such that $\mu(\cdot)$ has support over a finite interval were first given by B. Sz.-Nagy [6]. General necessary and sufficient conditions for a sequence to be of the form (1), with no restrictions on the support of $\mu(\cdot)$, were first given by J. S. MacNerney [4].

An Hermitian moment sequence (1) will be said to be determinate if $\mu(\cdot)$ is unique, and indeterminate otherwise (cf. Akhiezer [1], Dubois-Violette [3], and Shohat and Tamarkin [7]).

Main result. A determinate moment sequence $\left\{T_{j}\right\}$ can be "imbedded" in an indeterminate moment sequence on $\mathscr{K}=$ $\mathscr{H} \oplus \mathscr{H}$. For example, let $\left\{a_{j}\right\}_{1=0}^{\infty}$ be a scalar-valued indeterminate moment sequence, $I$ be the identity operator on $\mathscr{H}$, and $P$ be the orthogonal projection from $\mathscr{K}$ to $0 \oplus \mathscr{H}=\mathscr{H}$. The sequence, 


$$
T_{j}^{\prime}=\left(\begin{array}{cc}
a_{j} \cdot I & 0 \\
0 & T_{j}
\end{array}\right)
$$

is easily seen to be an indeterminate Hermitian moment sequence, while the compression of $\left\{T_{j}^{\prime}\right\}$ (cf. Putnam [5], pg. 76),

$$
P T^{\prime} P=T_{j}, \quad j=0,1,2, \cdots,
$$

is determinate. What is not at all obvious is that every indeterminate moment sequence on $\mathscr{H}$ can be imbedded in (that is, arises as a compression of) a determinate moment sequence on $\mathscr{H} \oplus \mathscr{H}$. The main purpose in writing this paper is to establish

THEOREM 1. (Imbedding Theorem) Let $T_{n}, \mu(\cdot)$ be as in (1) and let

$$
R_{j}=\int_{-\infty}^{\infty} t^{j} e^{-|t|} d \mu(t), \quad S_{j}=\int_{-\infty}^{\infty} t^{j} e^{-|t| / 2} d \mu(t)
$$

Then the sequence,

$$
\tilde{T}_{j}=\left(\begin{array}{ll}
R_{j} & S_{j} \\
S_{j} & T_{l}
\end{array}\right), \quad \quad j=0,1,2, \cdots
$$

defined on $\mathscr{K}=\mathscr{H} \oplus \mathscr{H}$, is a determinate Hermitian moment sequence.

The proof is postponed.

The Imbedding Theorem raises an interesting question: Let $\left\{T_{j}\right\}$ be a determinate moment sequence and let $P$ be the orthogonal projection onto a closed subspace, $\mathscr{H}_{p}$, of $\mathscr{H}$. Under what conditions will the compression of the moment sequence, $\left\{P T_{1} P\right\}$, be determinate?

For any given moment sequence, $\left\{T_{i}\right\}_{j=0}^{\infty}$, whether it is determinate or not, the subspace $\mathscr{H}_{p}$, will be said to be determinate or indeterminate depending on whether $\left\{P T_{j} P\right\}$ is determinate or indeterminate. A moment sequence for which every subspace is determinate will be called a completely determinate moment sequence. In general, a nontrivial characterization of the determinate subspaces of a moment sequence is not known. For completely determinate sequences, the problem is much simpler:

Lemma 1. A moment sequence of the form (1) is completely determinate if and only if it is determinate in all of the one dimensional subspaces of $\mathscr{H}$. A sufficient condition for this to occur is that

$$
\sum_{i=1}^{\infty}\left\|T_{2,}\right\|^{-1 / 2 j}=+\infty
$$


Proof. The necessity of the condition of being determinate in each one dimensional subspace follows from the definition. To establish sufficiency, let $\left\{T_{j}\right\}$ obey this condition, then for any $\phi \in \mathscr{H},\|\phi\|=1$, $\tau_{j}(\phi)=(T, \phi, \phi)$ is a determinate scalar moment sequence. If $\mu(\cdot)$ and $\hat{\mu}(\cdot)$ both generate $\{T\}$, then

$$
\tau_{j}(\phi)=\int_{-\infty}^{\infty} t^{j} d(\mu(t) \phi, \phi)=\int_{-\infty}^{\infty} t^{j} d(\hat{\mu}(t) \phi, \phi) .
$$

The determinacy of $\left\{\tau_{j}(\phi)\right\}$ then implies that for any Borel set $\Delta$,

$$
(\mu(\Delta) \phi, \phi)=(\hat{\mu}(\Delta) \phi, \phi) .
$$

Coupling (6) with the fact that $\mathscr{H}$ is a complex Hilbert space then implies $\mu(\Delta)=\hat{\mu}(\Delta)$. Hence, the full moment sequence is determinate.

Now, let $P$ be a projection onto a closed subspace, $\mathscr{H}_{p}$, of $\mathscr{H}$ and consider the corresponding one dimensional compressions of PT,P:

$$
\sigma_{J}(\phi)=\left(P T_{j} P \phi, \phi\right), \quad j=0,1,2, \cdots,
$$

where $\phi \in \mathscr{H},\|\phi\|=1$. From (7), it is clear that $\sigma_{j}(\phi)=0$ if $\|P \phi\|=0$ and

$$
\sigma_{I}(\phi)=\|P \phi\|^{2} \tau_{l}\left(\frac{P \phi}{\|P \phi\|}\right), \quad j=0,1,2, \cdots
$$

if $\|P \phi\| \neq 0$. In the former case, $\left\{\sigma_{J}(\phi)\right\}$ is obviously determinate. In the latter case, $\left\{\sigma_{l}(\phi)\right\}$ is determinate because, by (8), it is a positive constant multiple of a determinate moment sequence. A direct application of a Theorem of Hamburger (cf. Shohat and Tamarkin [7], p. 70) shows all such sequences are determinate. Thus, $\left\{P T_{j} P\right\}$ is determinate in each of its one dimensional subspaces and, by the preceeding discussion, is determinate. This, in turn, implies $\left\{T_{j}\right\}$ is completely determinate.

Finally, that (4) is a sufficient condition for $\left\{T_{\}}\right\}$to be completely determinate follows from the fact that for $\phi \in \mathscr{H},\|\phi\|=1$,

$$
\left|\tau_{l}(\phi)\right|=\left|\left(T_{j} \phi, \phi\right)\right| \leqq\left\|T_{l}\right\|, \quad j=0,1,2, \cdots .
$$

This, together with (4), implies $\left\{\tau_{j}(\phi)\right\}$ satisfies the ordinary Carleman condition (cf. [7], pg. 19) and is, therefore, determinate for each $\phi$. By the preceding discussion, $\left\{T_{j}\right\}$ is completely determinate. This ends the proof.

Condition (4) is certainly not a necessary condition for complete 
determinacy. Consider the following example (A. E. Nussbaum, private communication): Let $\left\{a_{j}\right\}$ be an indeterminate scalar (Hamburger) moment sequence and set

$$
\delta=\lim _{n \rightarrow \infty} \min _{x_{0}=1}\left(\sum_{j=0}^{n} \sum_{k=0}^{n} a_{j+k} x_{j} x_{j}\right)
$$

Then by the Theorem of Hamburger mentioned earlier (cf. [7], pg. 70), the sequence

$$
\begin{aligned}
& b_{0}=a_{0}-\delta \\
& b_{j}=a_{\jmath},
\end{aligned} \quad j=1,2,3, \cdots,
$$

is easily seen to be a determinate moment sequence. Moreover, since $\left\{a_{l}\right\}$ was indeterminate to begin with, it cannot satisfy the Carleman condition and, therefore, $\left\{b_{j}\right\}$ is a determinate moment sequence which does not satisfy the Carleman condition. Thus, the Carleman condition is not a necessary condition for determinacy, even in the scalar case.

The determinate moment sequence $\left\{b_{j}\right\}$ also has two other interesting properties, which can be demonstrated via the Theorem of Hamburger. The translates $\left\{b_{j+2 k}\right\}_{l=0}^{\infty}$ are also moment sequences, but they are indeterminate if $k \geqq 1$. Finally, adding the two determinate moment sequences $\left\{b_{\}}\right\}$and $\{\delta, 0,0, \cdots\}$ results in the indeterminate moment sequence $\left\{a_{l}\right\}$. Thus determinacy is not necessarily preserved when two determinate moment sequences are added.

Proof of Theorem 1. Since the positive $B(\mathscr{K})$-valued Borel measure,

$$
d \nu(t)=\left(\begin{array}{ll}
e^{-|t|} & e^{-|t| / 2} \\
e^{-|t| / 2} & 1
\end{array}\right) d \mu(t)
$$

generates the sequence $\left\{\tilde{T}_{l}\right\}$, it is a moment sequence. If $d \hat{\nu}(t)$ is any positive $B(\mathscr{K})$-valued Borel measure which generates $\left\{\tilde{T}_{\jmath}\right\}$, and if

$$
d \omega(t)=d \hat{\nu}(t)-d \nu(t)
$$

then

$$
\int_{-\infty}^{\infty} t^{j} d \omega(t)=0, \quad j=0,1,2, \cdots
$$

What needs to be shown is that $d \omega(t)=0$, for then $d \hat{\nu}=d \nu$ and $\left\{\tilde{T}_{l}\right\}$ is determinate. 
By considering vectors of the form $(\phi, 0)^{T} \in \mathscr{K}$, it is possible to show that the upper left component of $d \hat{\nu}(t), d \hat{\nu}_{1,1}(t)$, is a positive $B(\mathscr{H})$-valued Borel measure. Moreover, (12) implies that

$$
R_{l}=\int_{-\infty}^{\infty} t^{\prime} d \hat{\nu}_{1,1}(t)=\int_{-\infty}^{\infty} t^{\prime} e^{-|t|} d \mu(t)
$$

for $j=0,1,2, \cdots$. However, using the definition of $R_{j}$, the inequality

$$
\left\|R_{J}\right\| \leqq j^{\prime} e^{-j}\left\|T_{0}\right\|
$$

can be easily obtained. An application of the Carleman condition (4), given in Lemma 1 , then implies $R$, is completely determinate. This fact, coupled with (13), implies that the two measures in (13) are equal. Hence,

$$
d \omega_{1,1}(t)=0
$$

Again by considering vectors of a special form, $(\alpha \phi, \beta \phi)^{T}, \phi \in \mathscr{H}$, $\alpha, \beta \in \mathscr{C}$, it is clear that the $2 \times 2$ matrix-valued measures,

$$
\begin{aligned}
& d \hat{\nu}_{\phi}(t)=(d \hat{\nu}(t) \phi, \phi), \quad d \nu_{\phi}(t)=(d \nu(t) \phi, \phi), \\
& d \omega_{\phi}(t)=(d \omega(t) \phi, \phi),
\end{aligned}
$$

satisfy

$$
d \hat{\nu}_{\phi}(t) \geqq 0, \quad d \nu_{\phi}(t) \geqq 0, \quad \int_{-\infty}^{\infty} t^{i} d \omega_{\phi}(t)=0
$$

Fix $\phi \in \mathscr{H} . \quad B y(15)$ and (16),

$$
d \omega_{\phi}(t)=\left(\begin{array}{ll}
0 & d \sigma(t) \\
d \bar{\sigma}(t) & d \tau(t)
\end{array}\right)
$$

where $d \sigma(t)=\left(d \omega_{1,2} \phi, \phi\right), d \tau(t)=\left(d \omega_{2,2} \phi, \phi\right)$. Because the measures $d \hat{\nu}$ and $d \nu$ are bounded operator valued measures, all the measures $d \sigma$, $d \tau, d \mu_{\phi}(t)=(d \mu(t) \phi, \phi)$ are bounded and, respectively, are complex, real, and positive Borel measures. By the Radon-Nikodym Theorem, there exist $f(t), g(t) \in L^{1}\left(d \mu_{\phi}\right)$, and bounded Borel measures $d \tau_{s}(t)$, $d \sigma_{s}(t)$, singular with respect to $d \mu_{\phi}(t)$, such that

$$
\begin{aligned}
& d \tau(t)=f(t) d \mu_{\phi}(t)+d \tau_{s}(t) \\
& d \sigma(t)=g(t) d \mu_{\phi}(t)+d \sigma_{s}(t) .
\end{aligned}
$$


Combining (16), (18), and (19) gives

(20) $d \hat{\nu}_{\phi}(t)=\left(\begin{array}{ll}e^{-|t|} & e^{-|t| / 2}+g(t) \\ e^{-|t| / 2}+\overline{g(t)} & 1+f(t)\end{array}\right) \cdot d \mu_{\phi}(t)+\left(\begin{array}{ll}0 & d \sigma_{s}(t) \\ d \bar{\sigma}_{s}(t) & d \tau_{s}(t)\end{array}\right)$.

Let $\Delta$ be any subset of the real line such that $\mu_{\phi}(\Delta)=0$. Since $d \hat{\nu}_{\phi}(t) \geqq 0$,

$$
\hat{\nu}_{\phi}(\Delta)=\left(\begin{array}{ll}
0 & \sigma_{s}(\Delta) \\
\bar{\sigma}_{s}(\Delta) & \tau_{s}(\Delta)
\end{array}\right) \geqq 0
$$

The facts that trace and determinant of a nonnegative matrix are themselves nonnegative imply that

$$
\begin{aligned}
& \sigma_{s}(\Delta)=0 \\
& \tau_{s}(\Delta) \geqq 0 .
\end{aligned}
$$

Since the support of $\sigma_{s}$ is singular with respect to $d \mu_{s}(t)$, this implies that

$$
d \sigma_{s}(t)=0, \quad d \tau_{s}(t) \geqq 0 .
$$

To complete the proof of the theorem, it need only be shown that $f(t)=g(t)=0, d \tau_{s}(t)=0$. If this can be done, it follows from (20) that $d \hat{\nu}_{\phi}(t)=d \nu_{\phi}(t)$ for every $\phi$ in $\mathscr{H}$. Hence, for any Borel set $\Delta, \hat{\nu}_{\phi}(\Delta)=$ $(\hat{\nu}(\Delta) \phi, \phi)=\nu_{\phi}(\Delta)=(\nu(\Delta) \phi, \phi)$. Since the underlying space is a a complex Hilbert space, this is sufficient to imply that $\hat{\nu}(\Delta)=\nu(\Delta)$ and that $\left\{\tilde{T}_{j}\right\}$ is determinate.

To see that $g(t)=0$, first note that the matrix multiplying $d \mu_{\phi}(t)$ in (20) is nonnegative almost everywhere with respect to $\mu_{\phi}$. Otherwise, the integral of $d \hat{\nu}_{\phi}$ over a set which is both disjoint from the support of $\tau_{s}$ and has positive $\mu_{\phi}$-measure would be negative, which is impossible. Applying the condition that the determinant of a nonnegative matrix must itself be nonnegative and then multiplying the resultant inequality by $e^{|t|}$ yields

$$
\left|1+g(t) e^{\mid t / 2}\right|^{2} \leqq 1+f(t)
$$

Since both $1, f(t)$ belong to $L^{1}\left(d \mu_{\phi}(t)\right)$, the function $1+g(t) e^{|t| / 2}$ belongs to $L^{2}\left(d \mu_{\phi}(t)\right)$. But this in turn implies $g(t) e^{|t| / 2} \in L^{2}\left(d \mu_{\phi}(t)\right)$. Finally, this implies

$$
\int_{-\infty}^{\infty} e^{|t|}|g(t)|^{2} d \mu_{\phi}(t)=\int_{-\infty}^{\infty} e^{2|t|}|g(t)|^{2} e^{-|t|} d \mu_{\phi}(t)
$$


and

$$
e^{|t|} g(t) \in L^{2}\left(e^{-|t|} d \mu_{\phi}(t)\right)
$$

Combining (17), (18), (19), and (22) yields

$$
\int_{-\infty}^{\infty} t^{\prime} g(t) d \mu_{\phi}(t)=0=\int_{-\infty}^{\infty} t^{\prime} e^{|t|} g(t) e^{-|t|} d \mu_{\phi}(t) .
$$

Thus by (24) and (25), $e^{|t|} g(t)$ is in $L^{2}\left(e^{-|t|} d \mu_{\phi}(t)\right)$ and orthogonal to all polynomials. Since $\left\{R_{l}\right\}$ is a completely determinate moment sequence, $\left\{\left(R_{j} \phi, \phi\right)\right\}$ is a determinate moment sequence. The polynomials are therefore complete in $L^{2}\left(e^{-|t|} d \mu_{\phi}(t)\right)$ (cf. Akhiezer [1], pg. 45) and $e^{|t|} g(t)=0$. Hence,

$$
g(t)=0 \text { a.e. } \mu_{\phi} \text {. }
$$

It is now possible to show that $f(t)$ and $d \tau_{s}(t)$ are both 0 . Combining (20), (22), and (26), the measure $d \hat{\nu}_{\phi}$ has the form

$$
d \hat{\nu}_{\phi}(t)=\left(\begin{array}{cc}
e^{-|t|} & e^{-\mid t / 2} \\
e^{-|t| / 2} & 1+f(t)
\end{array}\right) d \mu_{\phi}(t)+\left(\begin{array}{cc}
0 & 0 \\
0 & d \tau_{s}(t)
\end{array}\right) .
$$

Again, it follows from the nonnegative character of the matrix multiplying $d \mu_{\phi}(t)$ that

$$
e^{-|t|} \leqq e^{-|t|}+e^{-|t|} f(t)
$$

and, hence,

$$
0 \leqq f(t), \quad \text { a.e. } \mu_{\phi}(\cdot)
$$

Now, however, because $d \hat{\nu}$ and $d \nu$ both generate the moment sequence $\left\{\tilde{T}_{f}\right\}$, it is obvious that

(29)

$$
\begin{aligned}
(T, \phi, \phi) & =\int_{-\infty}^{\infty} t^{\prime}(1+f(t)) d \mu_{\phi}(t)+\int_{-\infty}^{\infty} t^{\prime} d \tau_{s}(t) \\
& =\int_{-\infty}^{\infty} t^{j} d \mu_{\phi}(t) .
\end{aligned}
$$

From (29), it follows that for $j=0$,

$$
\int_{-\infty}^{\infty} f(t) d \mu_{\phi}(t)+\int_{-\infty}^{\infty} d \tau_{s}(t)=0 .
$$


By (25), (28), and (30),

$$
f(t)=0, \quad \text { a.e. } \mu_{\phi}, \quad d \tau_{s}=0,
$$

and, by the remarks made earlier, $\left\{\tilde{T}_{j}\right\}$ is a determinate moment sequence.

Reducing subspaces. If an Hermitian moment sequence, $\left\{T_{j}\right\}$, is determinate and can be expressed as

$$
T_{j}=U_{j}+V_{i}, \quad j=0,1,2, \cdots,
$$

where $\left\{U_{j}\right\}$ and $\left\{V_{j}\right\}$ are also Hermitian moment sequences, then both sequences are determinate (the converse is, of course, false - see the remarks following Lemma 1). If this were not the case and, say, $\left\{U_{j}\right\}$ were generated by both $d \rho(t)$ and $d \hat{\rho}(t)$, then if $d \sigma(t)$ generates $\left\{V_{j}\right\}$, both $d \rho(t)+d \sigma(t)$ and $d \hat{\rho}(t)+d \sigma(t)$ would generate $\left\{T_{j}\right\}$. Since $\left\{T_{j}\right\}$ is assumed determinate, these two measures are equal. This implies $d \hat{\rho}(t)=d \rho(t)$, and $\left\{U_{j}\right\}$ is determinate. These considerations lead to

THEOREM 2. If $\left\{T_{j}\right\}$ is a determinate Hermitian moment sequence, $P$

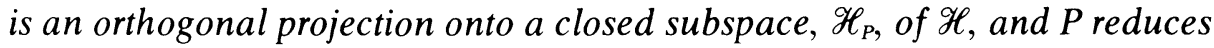
$\left\{T_{j}\right\}$,

$$
P T_{j}=T_{j} P=P T_{j} P, \quad j=0,1,2, \cdots,
$$

then both $\left\{P T_{j} P\right\}$ and $\left\{(I-P) T_{j}(I-P)\right\}$ are determinate and $\mathscr{H}_{P}, \mathscr{H}_{I-P}$ are determinate subspaces of $\mathscr{H}$ with respect to $\left\{T_{j}\right\}$.

Proof. Note that,

$$
T_{j}=P T_{j} P+(I-P) T_{j}(I-P), \quad j=0,1,2, \cdots .
$$

Both terms on the right are Hermitian moment sequences. Since $\left\{T_{j}\right\}$ is assumed determinate, both sequences are, by the discussion given above, determinate. The rest of the theorem follows from the definition of determinate subspace.

It should be noted that the theorem just proved, along with the Imbedding Theorem, show that subspaces of indeterminacy are associated with "off-diagonal" elements being present when a $2 \times 2$ matrix representation is used.

ACKNOWLEgDements. The author gratefully acknowledges the help of J. S. MacNerney, A. E. Nussbaum, and S. Slinker. 


\section{REFERENCES}

1. N. I. Akhiezer, The Classical Moment Problem, Hafner Publishing Co., New York, 1965.

2. S. K. Berberian, Notes on Spectral Theory, D. van Nostrand, Princeton, 1966.

3. M. Dubois-Violette, A generalization of the classical moment problem on *-algebras with applications to relativistic quantum theory. I, Commun. Math. Phys., 43 (1975), 225-254.

4. J. S. MacNerney, Hermitian moment sequences, Trans. Amer. Math. Soc., 103 (1962), 45-81.

5. C. R. Putnam, Commutation Properties of Hilbert Space Operators, Springer, New York, 1967.

6. B. Sz.-Nagy, A moment problem for self-adjoint operators, Acta Mathematica (Hung.), 3 (1952), 285-292.

7. J. A. Shohat and J. D. Tamarkin, The Problem of Moments, Amer. Math. Soc., New York, 1939.

Received April 2, 1976. This work is partly supported by NSF Grant No. MCS76-06631.

TEXAS A \& M UNIVERSITY

College, TX 77843 



\section{PACIFIC JOURNAL OF MATHEMATICS}

\section{EDITORS}

RICHARI) ARENS (Managing Editor)

University of California

Los Angeles, CA 90024

R. A. Beaumiont

University of Washington

Seattle, WA 98105

\section{J. DugunduI}

Department of Mathematics University of Southern California Los Angeles, CA 90007

D. Gilbarg and J. Milgram Stanford University

Stanford, CA 94305

\section{ASSOCIATE EDITORS}

E. F. BECKENBACH
B. H. NEUMANN

F. Wolf

K. YoshidA

\section{SUPPORTING INSTITUTIONS}

UNIVERSITY OF BRITISH COLUMBIA CALIFORNIA INSTITUTE OF TECHNOLOGY

UNIVERSITY OF CALIFORNIA

MONTANA STATE UNIVERSITY

UNIVERSITY OF NEVADA

NEW MEXICO STATE UNIVERSITY

OREGON STATE UNIVERSITY

UNIVERSITY OF OREGON

OSAKA UNIVERSITY

\author{
UNIVERSITY OF SOUTHERN CALIFORNIA \\ STANFORD UNIVERSITY \\ UNIVERSITY OF HAWAII \\ UNIVERSITY OF TOKYO \\ UNIVERSITY OF UTAH \\ WASHINGTON STATE UNIVERSITY \\ UNIVERSITY OF WASHINGTON \\ AMERICAN MATHEMATICAL SOCIETY
}

The Supporting Institutions listed above contribute to the cost of publication of this Journal, but they are not owners or publishers and have no responsibility for its contents or policies.

Mathematical papers intended for publication in the Pacific Journal of Mathematics should be in typed form or offset-reproduced (not dittoed), double spaced with large margins. Underline Greek letters in red, German in green, and script in blue. The first $p: 1<$ graph or two must be capable of being used separately as a synopsis of the entire paper. Items of the biblography should not be cited there unless absolutely necessary, in which case they must he identified by author and Journal, rather than by item number. Manuscripts, in duplicate, may be sent to any one of the four editors. Please classify according to the scheme of Math. Reviews, Index to Vol. 39. All other communications should be addressed to the managing editor, or Elaine Barth, University of California, Los Angeles, California, 90024.

100 reprints are provided free for each article, only if page charges have been substantially paid. Additional copies may be obtained at cost in multiples of 50 .

The Pacific Journal of Mathematics is issued monthly as of January 1966. Regular subscription rate: $\$ 72.00$ a year (6 Vols., 12 issues). Special rate: $\$ 36.00$ a year to individual members of supporting institutions.

Subscriptions, orders for back numbers, and changes of address should be sent to Pacific Journal of Mathematics, 103 Highland Boulevard, Berkeley, California, 94708.

PUBLISHED BY PACIFIC JOURNAL OF MATHEMATICS, A NON-PROFIT CORPORATION Printed at Jerusalem Academic Press, POB 2390, Jerusalem, Israel.

\section{Copyright (C) 1976 Pacific Journal of Mathematics} All Rights Reserved 


\section{Pacific Journal of Mathematics}

\section{Vol. 66, No. 2 December, 1976}

Gerald A. Beer, Tax structures whose progressivity is inflation neutral..... 305

William M. Cornette, A generalization of the unit interval............. 313

David E. Evans, Unbounded completely positive linear maps on

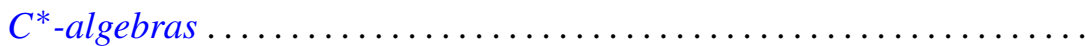

Hector O. Fattorini, Some remarks on convolution equations for

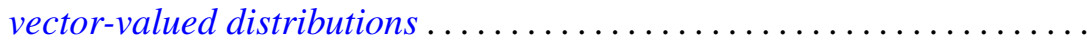

Amassa Courtney Fauntleroy, Automorphism groups of unipotent groups of

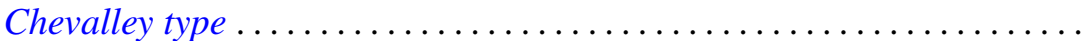

Christian C. Fenske and Heinz-Otto Peitgen, On fixed points of zero index in

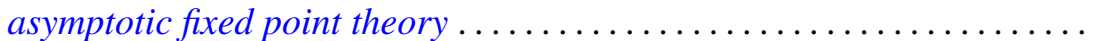

Atsushi Inoue, On a class of unbounded operator algebras. II ............

Herbert Meyer Kamowitz, The spectra of endomorphisms of algebras of

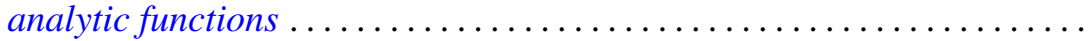

Jimmie Don Lawson, Embeddings of compact convex sets and locally compact cones ....................................

William Lindgren and Peter Joseph Nyikos, Spaces with bases satisfying certain order and intersection properties .....................

Emily Mann Peck, Lattice projections on continuous function spaces ...... 477

Morris Marden and Peter A. McCoy, Level sets of polynomials in $n$ real variables...

Francis Joseph Narcowich, An imbedding theorem for indeterminate Hermitian moment sequences......................

John Dacey O'Neill, Rings whose additive subgroups are subrings ...

Chull Park and David Lee Skoug, Wiener integrals over the sets bounded by sectionally continuous barriers .....................

Vladimir Scheffer, Partial regularity of solutions to the Navier-Stokes equations.

Eugene Spiegel and Allan Trojan, On semi-simple group algebras. II

Katsuo Takano, On Cameron and Storvick's operator valued function space integral 